\title{
17th International Symposium on Analytical Ultracentrifugation and Hydrodynamics
}

\author{
Alexandra S. Solovyova
}

Received: 14 November 2008 / Revised: 12 December 2008 / Accepted: 2 January 2009 / Published online: 3 February 2009

(C) European Biophysical Societies' Association 2009

Analytical ultracentrifugation (AUC) began its history as a method for studies of biological molecules in the 1920s. During the past decade, AUC has undergone a real renaissance due to the development of high precision and sensitive instrument (currently equipped with up to three types of detection), and the advances in sedimentation data analysis. AUC has become a dominant technique amongst other biophysical methods for the characterisation of biomolecules and their interactions under physiologically relevant conditions. At present, AUC brings together scientists working in the areas of physics, computing, biology and chemistry. As a result, the scientific community involved in AUC use and development is constantly growing, and the exchange of ideas and contacts between users and developers has become an important part in the progression of this field.

The 17th International Symposium on Analytical Ultracentrifugation and Hydrodynamics was held at Newcastle upon Tyne, UK (hosted by the Institute for Cell and Molecular Biosciences, University of Newcastle) on 11-12 September 2008. The satellite AUC workshop (8-10 September 2008) was organised before the meeting to demonstrate to the participants the latest achievements in AUC data interpretation, hydrodynamic modelling and instrument diagnostics. The event attracted 75 academic scientists with

\footnotetext{
A. S. Solovyova $(\square)$

Faculty of Medical Sciences,

Institute for Cell and Molecular Biosciences,

Newcastle University, Framlington Place,

Newcastle upon Tyne NE2 4HH, UK

e-mail: alexandra.solovyova@ newcastle.ac.uk
}

wide range of expertise and scientific representatives from pharmaceutical, chemical and analytic industries (Fig. 1). The content of the meeting was driven by the current activities in AUC user's community. Therefore the combination of the traditional subjects in the area such as (1) instrument and data analysis development; (2) AUC applications for biological systems and (3) AUC applications for colloids and polymers with the latest advances in hydrodynamic theory and cross-method interpretation (for example, complementary applications of AUC and small-angle X-rays scattering, light scattering) gave the complete picture of today's advances in AUC and hydrodynamics.

The current special issue of the European Biophysical Journal illustrates the whole spectrum of AUC-related subjects. As some examples, the followings could be listed: the development of open source platform for AUC (The Open AUC Project) (Langhorst, Laue, Demeler and Cölfen); AUC data analysis software development represented by Arthur Rowe, Borries Demeler, Mattia Rocco and Joachim Belhke; the latest advances in hydrodynamic bead modelling theory such as hydrodynamic modelling of multi-subunit structures and calculation of intrinsic viscosity of bead models (Garcia de la Torre et al.); macromolecule conformation modelling and related hydrodynamic calculations (Durshchlag and Zipper); thermodynamic approaches in AUC illustrated by a novel variant of the EdelsteinSchachman method for determination of macromolecule partial specific volume, particularly well suited for study of polyelectrolytes such as DNA and RNA (Hellman and Fried) and second virial analysis (Scott et al). Biological applications are represented by the study of aggregation processes of amyloid- $\beta$ peptide in the presence of designed $\beta$-sheet breaker (Nagel-Steger et al.), and low-resolution structural studies of pili subunits from Streptococcus pyogenes (Solovyova et al.). 


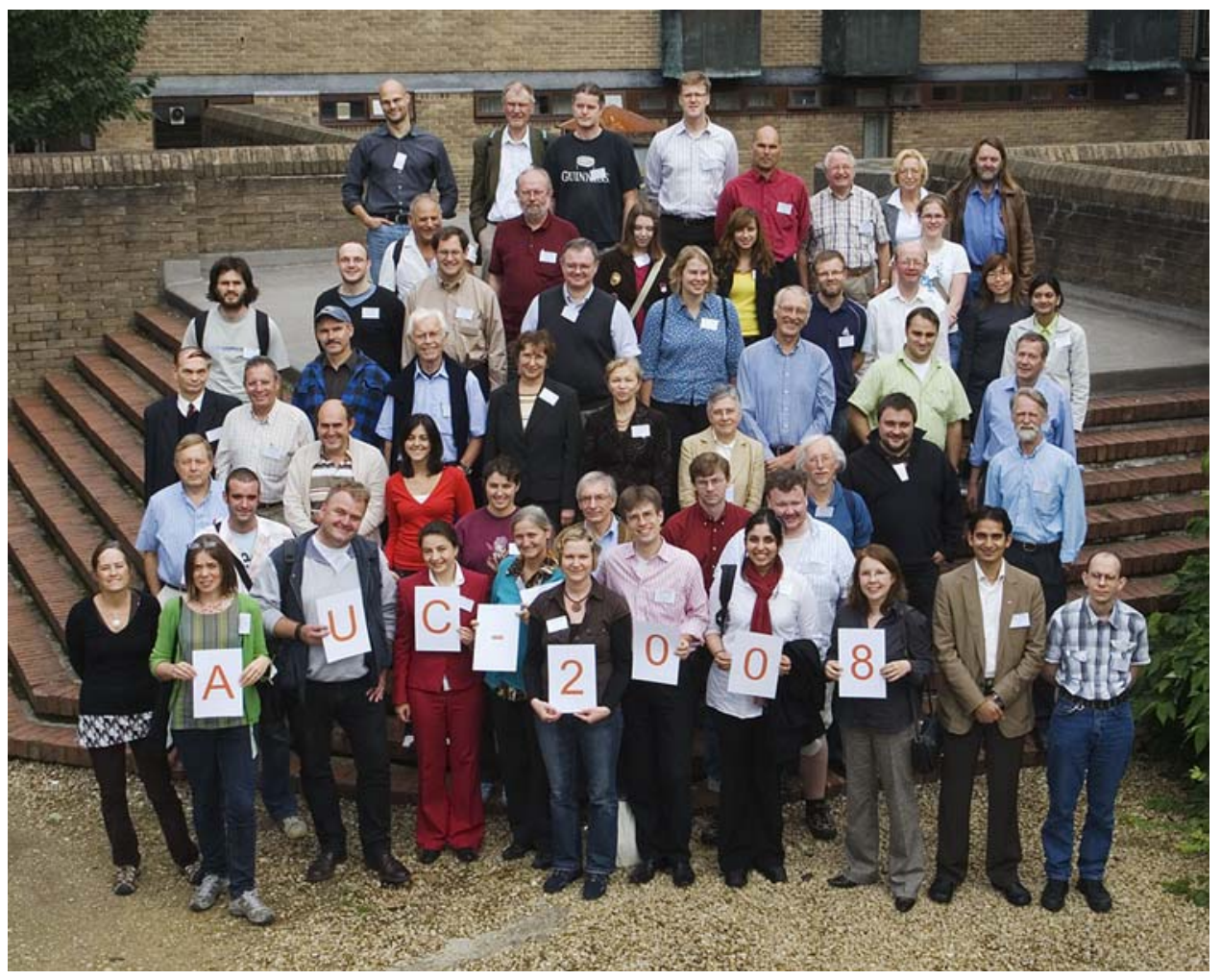

Fig. 1 AUC\&HYDRO-2008 (Newcastle, 8-12 September 2008) symposium and workshop participants listed from bottom to top: first row (from left to right): Karen Fleming, Olwyn Byron, Helmut Coelfen, Alex Solovyova, Christine Ebel, Antje Voelkel, Wendel Wohleben, Swetha Vijayakrishnan, Luitgard Nagel-Steger, Vikas Mittal, Nicola Wenta. Second row (from left to right) Walter Stafford, Diego Amoros, Carlos Alfonso, Ariadna Martos-Sanchez, Aline Appourchaux, Francis Mueller, Chad Brautigam, David Scott, Mattia Rocco. Third row (from left to right) Helmut Durchshlag, José Garcia de la Torre, Borries Demeler, Joachim Belhke, Natalia Chebotareva,
Elena Tarabukina, Erika Schmidt, Bertrand Raynal, Curt Ekström. Fourth row (from left to right) Engin Karabudak, Emmanuel Risse, Glen Ramsay, Mike Fried, Ute Curth, Georges Pavlov, Steffano Bruzzano, Eckhard Goernitz. Fifth row (from left to right) Ariel Lustig, Claus Urbanke, Natalie Naue, Sirine El Houry, Gordon Morris, Andrew Leech, Shirley Ang, Kurchid Asma. Sixth row (from left to right) Holger Strauss, Dieter Lechner, Niels Volkmann, Anthony Curran, Kavin Jackson, Arthur Rowe, Christine Wandrey, Jacqueline Marchall, Richard Thomas 